\section{Inflammation memory}

Contact sensitizers elicit a population of natural killer (NK) cells with memory-like functions that reside in the liver. In Immunity, Hornung and colleagues use a model of contact hypersensitivity to show that the establishment of NK cell memory is dependent on the NLRP3 inflammasome. The application of monobenzone onto the skin drives an NK cell-mediated cytotoxic immune response to melanocytes. The hapten-induced NK cells reside in the liver and can transfer long-term monobenzene-specific responses to naive mice. Monobenzone induces the infiltration of macrophages into the skin as well as the recruitment of macrophages with a tissue-resident phenotype to the lymph nodes, and the infiltration of macrophages and NK cells into the skin is dependent on NLRP3 and interleukin 18. Activation of inflammasomes in tissue-resident macrophages is required for both the priming phase and the recall phase of monobenzone-induced memory NK cell responses.

Immunity (7 June 2016) doi:10.1016/j.immuni.2016.05.008

\section{The protective crypt}

Intestinal epithelial stem cells reside at the base of intestinal crypts. In Cell, Kaiko et al. identify the microbial metabolite butyrate as an inhibitor of intestinal stem-cell proliferation. Butyrate is a product of bacterial fermentation of dietary fiber. At concentrations found in the mouse colon (1-5 mM), butyrate suppresses epithelial-cell proliferation in vitro and in the crypt-less zebrafish colon or after injuryinduced exposure of stem cells in the mouse colon. This inhibitory effect is reversed by culture with differentiated colon epithelial cells, which metabolize butyrate as an energy source. Mice that lack the enzyme that converts butyrate to acetyl-CoA have less proliferation of colonic epithelial cells. Butyrate inhibits histone-deacetylase activity, induces alterations to gene expression and increases binding of the transcription factor Foxo3 to the promoters of the genes encoding the cell-cycle regulators Cdkn1a, Cdk1c and Gadd45b in intestinal stem cells. In vivo, Foxo inhibitors 'rescue' the effect of butyrate on epithelial-cell proliferation.

Cell (2 June 2016) doi:10.1016/j.cell.2016.05.018

\section{Prospecting TCRs for immunotherapy}

Environmental stress can influence anti-tumor immune responses. In Cancer Immunology Research, Xiao et al. identify a positive role for the neuropeptide BDNF in eliciting enhanced cytotoxic $\mathrm{CD}^{+} \mathrm{T}$ cells directed against melanoma tumors. Mice living in socially stimulating environments express more BDNF. Both the sympathetic nervous system and the hypothalamic-pituitary-adrenal axis contribute to the effects mediated by BDNF. Enhanced hypothalamic expression of BDNF increases the frequency of anti-tumor $\mathrm{CD} 8^{+} \mathrm{T}$ cells in lymph nodes and diminishes the tumor burden, whereas suppression of BDNF expression produces the opposite effect. These findings indicate a role for neuralimmunological communication that can lead to better control of tumor progression.

$L A D$

Cancer Immunol. Res. 4, 488-497 (2016)

\section{Dengue suppresses MAVS}

Viral infection triggers the production of interferons; however, dengue virus can suppress this antiviral response. In the Journal of Virology, He et al. show that the dengue virus non-structural protein NS4a subverts induction of the production of type I interferons by interfering with the RIG-I-MAVS activation pathway. The recognition of viral RNA by the RNA helicase RIG-I triggers interactions between the caspase-recruitment domains of RIG-I and those of the signaling adaptor MAVS that lead to activation of kinase TBK1 and the expression of genes encoding antiviral products, including Ifnb, mediated by the transcription factor IRF3. NS4a potently disrupts this interaction by binding to the MAVS caspase-recruitment and carboxyl-terminal domains and thereby prevents Ifnb expression. NS4a localizes to the mitochondrial membrane, and the transmembrane domain 3 can mediate inhibition of MAVS. These findings suggest targeting NS4a or its ability to interact with MAVS might enhance innate antiviral responses to dengue.

J. Virol. (1 Jun 2016) doi:10.1128/JVI.00221-16

Written by Laurie A. Dempsey, Zoltan Fehervari \& Ioana Visan
The elimination of tumors through vaccination or the adoptive transfer of tumor-specific T cells is an attractive option but is technically challenging because of difficulties in raising robust and appropriate immune responses. In Science, Schumacher and colleagues study patients with melanoma and find that their endogenous anti-tumor responses are poor, most probably due to immunoediting. Therefore, the authors turn to healthy donors as a potential source of reactivity of the $\mathrm{T}$ cell antigen receptor to melanoma neoantigens. An HLA-binding screen of potential melanoma neopeptides reveals a much greater number of healthy donor $T$ cells that recognize melanoma. Many of these donor-derived $\mathrm{T}$ cell antigen receptors can be successfully introduced into recipient $T$ cells, which can result in specific recognition of tumor neoantigens. These findings reveal that donors can act as a rich source of neoantigen reactivity, and this could inform their use in adoptive cancer immunotherapy.

Science 352, 1337-1341 (2016)

\section{A Nod to type 1 diabetes}

Type 1 diabetes (T1D) is triggered by both genetic factors and environmental factors, and among the latter, the gut microbiota seems to be important. In the Journal of Experimental Medicine, Carlos and colleagues use a streptozotocin-induced model of T1D to investigate the importance of the gut microbiota. Treatment with streptozotocin leads to the translocation of bacteria to pancreatic lymph nodes and results in the production of inflammatory cytokines by macrophages and dendritic cells. Critically, the induction of these cytokines depends on expression of the intracellular bacterial sensor Nod2. Accordingly, treatment with broad-spectrum antibiotics or Nod2 deficiency in mice results in relative protection from T1D. These data reveal a mechanism by which breakdown of the gut barrier can initiate a cascade that leads to the manifestation of T1D.

J. Exp. Med. (20 June 2016) doi:10.1084/jem.20150744 